\title{
Exploring Users' Attitudes towards Social Interaction Assistance on Google Glass
}

\author{
Qianli Xu, Michal Mukawa, Liyuan Li, Joo Hwee Lim, Cheston Tan, \\ Shue Ching Chia, Tian Gan, Bappaditya Mandal \\ Institute for Infocomm Research, Agency for Science, Technology and Research \\ 1 Fusionopolis Way, \#21-01 Connexis (South Tower), Singapore \\ $\{$ qxu, stumam, lyli, joohwee, cheston-tan, scchia, gant, bmandal\}@i2r.a-star.edu.sg
}

\begin{abstract}
Wearable vision brings about new opportunities for augmenting humans in social interactions. However, along with it comes privacy concerns and possible information overload. We explore users' needs and attitudes toward augmented interaction in face-to-face communications. In particular, we want to find out whether users need additional information when interacting with acquaintances, what information they want to access, and how they use it. Based on observations of user behaviors in interactions assisted by Google Glass, we find that users in general appreciated the usefulness of wearable assistance for social interactions. We highlight a few key issues of how wearable devices affect user experience in social interaction.
\end{abstract}

\section{Author Keywords}

Social interaction; Google Glass; wearable vision; social context; privacy.

\section{ACM Classification Keywords}

H.5.2 Information Interfaces and Presentation: User Interfaces - interaction styles; H.1.2 Models and Principles: User/Machine Systems - human factors.

\section{INTRODUCTION}

Communicating with acquaintances involves high-level social cognition, such as knowledge of the target person, understanding of social settings and etiquette, and the purpose and expectations of other persons. While most people are able to communicate effectively without external support, there are many occasions when certain kind of communication assistance is desirable, e.g. when one forgets the name of another person [5, 13], or meets a stranger whose personal information is unknown [3]. In these situations, a piece of relevant information about others may prevent possible diversion or offense.

(C) 2015 Association for Computing Machinery. ACM acknowledges that this contribution was authored or co-authored by an employee, contractor or affiliate of a national government. As such, the Government retains a nonexclusive, royalty-free right to publish or reproduce this article, or to allow others to do so, for Government purposes only.

$A H^{\prime} 15$, March 09 - 11, 2015, Singapore, Singapore

Copyright 2015 ACM 978-1-4503-3349-8/15/03 ...\$15.00

http://dx.doi.org/10.1145/2735711.2735831
A basic form of interaction assistance is prosthetic memory typically implemented on mobile devices [6]. However, the usage of prosthetic memory has been scant because of its limited functionality, and the distractions it causes to an ongoing conversation. A number of methods and systems have been proposed to provide information to users with certain mental disorders [7, 10-12]. However, they barely provide real-time assistance in the communications.

Wearable solutions, e.g. Google Glass, make it technically possible to provide instant information in routine communications. In particular, information can be retrieved and displayed without occupying users' full attention or requiring overt control of the device. However, personal assistance in the realm of social interactions is extremely tricky. At the personal level, such a technology may engender a negative self-image of being dependent on "prosthetic intelligence". At the interpersonal level, the additional information load may cause attention deficit. Considering the dynamics of face-to-face communications, such attention deficit may lead to discontinuity of the conversation and infringement of social etiquette [2]. At the social level, a persistent fear has been the disclosure of privacy information and potential misuse of it [4]. When the personal identity becomes transparent in the Glass, privacy concerns become a paramount issue.

In view of the possibilities provided by wearable vision and the mixed feelings regarding social interaction assistance, we want to investigate whether people have a need for social interaction assistance from a wearable system, what information users want to get from it, what factors influence their attitudes towards such a system, and how they perceive the social implications of the technology. To do so, we develop a wearable system on Google Glass that serves as an external memory and provides biographical information of another person (e.g. an acquaintance). Thus, it augments humans in their social interactions. The system is used in an experiment involving face-to-face interactions between multiple persons. By analyzing subjects' feedback in a semi-structure interview, we try to understand users' experience in the interactive process. We report qualitative results on information content, visualization, and interactivity. We are the first to explore users' needs and attitudes towards interaction assistance using a wearable device, i.e. Google Glass, in face-to-face communications. 
Evaluation of the user feedback leads to useful implications to augmenting humans in social interactions.

\section{USE CASE}

We are aware that people with normal mental capacity are capable of communicating with others without external assistance. However, we want to explore potential benefits of augmented memory in a few plausible applications. For example, in a conference socializing session, the system may recognize persons who are of interest to a wearer and provide relevant information, such as name, affiliation, research interests, etc. In this study, we adopt a use case where a new staff meets with his/her colleagues in a company. In this situation, there is an apparent need for the new staff to get to know their 'coworkers', to build up relationships, and to complete collaborative tasks.

\section{SYSTEM}

Our system comprises a Google Glass connected with an Android smartphone via Bluetooth. The image processing functions are implemented on the smartphone, including scene recognition (i.e. locations, such as meeting rooms, corridors, restaurants, etc.) and face recognition [9]. When a matching face is found in the database (stored locally on the smartphone), the system displays (on the screen of Google Glass) context-related information about the person. It should be noted that one could adopt hardcoded information retrieval without face recognition in a small-scale experiment. We chose to develop a working prototype with face recognition capacity to simulate technical uncertainties in practical scenarios. We included 28 items of biographical information that are categorized into 6 groups, namely, work, personal, education, social, leisure, and family. In addition, we include an "All" category, which contains all information items of a person in alphabetical order.

A sample UI on Google Glass is shown in Figure 1 (right). The top-right image shows the UI for capturing the human face. The bottom-right image displays the retrieved information of the person. The portrait photo and the text under it (i.e. name, position, and company) are always visible. A user can navigate between categories by swiping back and forth on the touchpad, and browse displayed items in a category by swiping up and down, if the category contains more than three items.

In addition, we build an alternative system on a standalone mobile phone (HTC M8) with similar functionalities. In the phone version, a face image is captured by the phone camera and the UI is displayed on the phone screen. Normal touch operations on the screen are adopted to control the phone. Other than these, the functionalities are identical in the two systems. Figure 1 (left) shows the UI of the mobile phone version. The experiment adopts the mobile phone as a benchmarking system for the following considerations. While both devices are easily accessible, they are characterized by different interaction modalities, such as display size, control mechanisms, and body posture

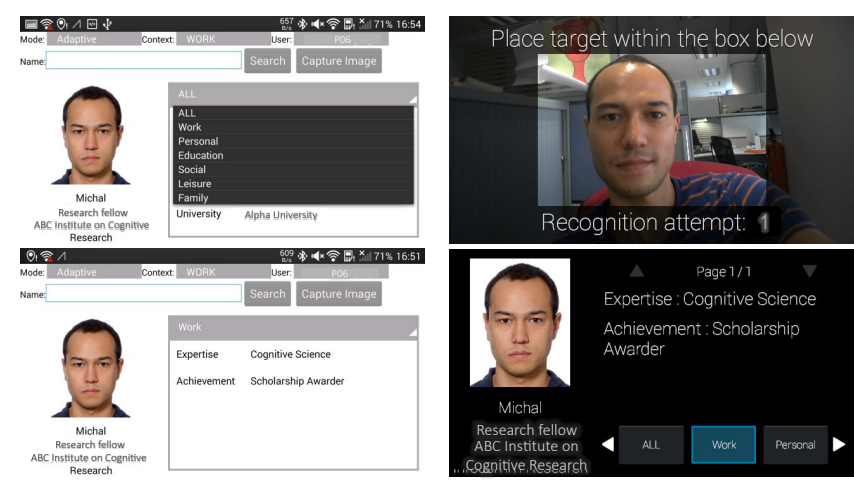

Figure 1. Personal information displayed on the mobile phone (left) and Google Glass (right).

in image capturing. Another important difference lies in users' familiarity with these two devices.

\section{EVALUATION}

\section{Procedure}

The system is evaluated in a user study requiring subjects to engage in two sessions of interaction. Each experiment session involved one or two subjects and at least two staff members from the host organization. The subjects did not know each other and they did not know the staff. There were two meeting segments arranged for the subjects, including a formal meeting in a meeting room and a casual conversation in a pantry. Each segment lasted for about 10 minutes. The subjects were instructed to consider a situation where they just started working in the host organization and joined a team of 10 staff. By talking to the staff in two brief segments, they needed to meet two requirements: (1) to get to know their new "colleagues", and (2) to actively contribute to planning a team-building event. In the second task, they needed to discuss about three team-building options and select one that fits the interests of their "colleagues". The order of the meeting segments (i.e., the venue of the meeting) was randomized and counter-balanced against device usage. Two subjects used the same type of device in the same meeting segment. The meeting procedure was recorded using digital video camera for further analysis. A semi-structured interview was conducted after two meeting segments. In the interview, we inquired about user's feedback on various aspects of system usage, such as the content of personal information, the layout of information display, how they used the system, what mentalities they had when using the device, etc.

\section{Participants}

We recruited 20 subjects (13 male; mean age: 24.6 yrs, STD 5.2 yrs) and randomly assigned them into 11 experiment sessions ( 9 sessions hosting 2 subjects, and 2 sessions hosting 1 subject, respectively). For the two consecutive meeting segments in an experiment, at least one staff member was changed. If there was an unchanged member, he/she did not take the initiative to introduce the new member. This is intended to ensure that the subject had an incentive to use the device to recognize the new person. 


\section{RESULTS}

The subjects' feedback was analyzed to derive meaningful themes of user attitudes. In so doing, we focused on the system functionality and their implications to the user experience along three dimensions.

\section{Information content}

It refers to what (personal) information is provided to the user. Since the information content is identical between the mobile phone and Glass-based system, users did not hold different opinions in this aspect. The subjects generally considered the current content of personal information appropriate. It was comprehensive enough to cover most aspects of a person.

Further, we want to find out if the subjects have used the provided information to initiate topics of conversation. More than half of the subjects said they have used the retrieved information to find a topic for discussion. However, they seldom did so immediately after reading certain information on the display. This is because they felt it awkward to change a topic amid an on-going conversation. Certain information was particularly interesting for topic finding, namely commonality between the user and the staff member, especially about their hobbies. This agrees with the classical social paradigm [8], which suggests that similarity in activities and attitudes is predictive of communication inclinations.

With respect to the self-reported topic-finding activities using the interaction assistance, there seems to be notable skepticisms, as mentioned by P5: "It is rather creepy if I tell someone - 'I know you like baking". Such an act was considered intrusive and socially indecorous. Inappropriate usage of such information for topic-finding may naturally cause a fear of being peeked at, and lead to uneasiness of both parties. Some factors may mitigate such a feeling, e.g. when the two parties know each other to a certain extent, and when they are aware of and agree to use the device. Similar findings was reported in applications of augmented reality [1]. In addition, it is advisable to start a topic in a less intrusive way. For example, instead of directly inquiring about others' personal lives, one may choose to self-disclose - In my spare time, I cook a lot. Or one can wait for a more appropriate opportunity to start the topic, as already did by some subjects. Another psychological barrier was that users might be conscious of a negative self-image if they relied too much on a device for discussion topics. P9, who considered himself to be fairly outgoing, said "...others may think I'm very shy if I find a topic like that". In view of these issues, one may need to use certain tactics to find discussion topics using information from the device.

\section{Information visualization}

It refers to how the personal information is presented. With different viewing position, orientation, display size and resolution, the two devices provoked divergent opinions. Users were generally satisfied with the UI design on the
Glass. They were able to understand the structure of information and the logic of touch operations with minimal training. As such, the UI design seems to be effective for personal information display.

The mobile phone, on the other hand, was appreciated for providing a better overview of the information structure, which in turn enable more efficient information search and browsing. However, users expected to read more detailed information on the mobile phone beyond a few key words. This is logical because we did not customize the content of the information for optimal display on the mobile phone.

Most users preferred head-up viewing experience on Google Glass. Only two subjects were in favor of the mobile phone due to familiarity. The viewing experience seemed to have significant influence on user behaviors and social awareness. Apparently, they were more reluctant to lower their head and check information on the mobile phone than to eye-up at the Glass display. P2 mentioned " $I t$ is rather rude if I bow down and look at the phone, especially when talking to someone of higher position."

However, even the less resource-demanding Glass display caused attention deficit, and in turn notable social consciousness. A number of subjects reported difficulties in following an on-going conversation when they read the text on the Glass display. P4 said 'I was totally lost. I don't know what the others were talking about". Some users appeared to be more positive about divided attention, as P11 put "I'm pretty okay with multitasking. Just that I need time to get used to it". Nevertheless, a more prevalent concern has been the possible misunderstanding of the conversation partner - "If your eyes always go about, people may think you are not listening or have no interest" [P18]. Moreover, there were reported difficulties in maintaining eye contact. In many occasions, reading information on the Glass display even for a short period of time caused loss of eye contact. Therefore, many subjects tried to avoid reading information when a conversation was going on, especially when there was eye contact. It seems worthwhile to optimize the timing of information display to avoid blocking the natural eye contact.

\section{Interactivity}

It means how a user operates the system, and how the system responds to the user. Operating on a mobile phone was straightforward and considered easy for all the subjects. Nevertheless, the function of search by face image provoked apparent concerns because pointing the phone camera at a person's face seemed rather rude. That is the reason why a few subjects mentioned that they would rather do a name search under the table. However, a consensus was that under-table phone viewing was by no means a good remedy - it was considered impolite to manipulate the mobile phone, openly or secretly.

In comparison, browsing information on Google Glass by tapping or swiping was less obtrusive, although it does not 
fare much better in front of an acquaintance. The hand movement beside the face and eye was noticeable and distracting. Subjects felt it disruptive to the conversation because others might feel offended or misunderstand it as a sign of confusion or lack of interest.

\section{DISCUSSIONS}

From the subjects' feedback, we find that Google Glass does provide better support to information seeking in social interactions as compared to a mobile phone. While the novelty effect and curiosity might have contributed to a higher preference level, a less momentous psychological barrier probably played a role when users can take a chance to elude from a conversation for quick information viewing.

However, we observed minimal effect of the Glass in effectuating deeper user engagement in the interactions. It did not help much to incur conversational topics, nor did it foster rapport between the subjects and the conversation partners. There are multivariate factors for this observation. First, the duration of the interactions was quite short, making it difficult for a subject to fully exploit the functionalities of the system and the benefits it may provide. Second, the scope of interactions was confined to a few predefined tasks. While this was necessary to ensure experiment controllability, it might have hindered the subjects' motivation to engage deeper with other parties. Third, being a working prototype, the system was not robust and comprehensive enough to fully support the social interaction assistance in the wild. Future work will be carried out based on improved system performance in longitudinal studies.

\section{CONCLUSION}

We investigated users' needs and attitudes towards social interaction assistance on wearable devices. We built the first working prototype of social interaction assistance on Google Glass, which allows a wearer to retrieve personal information of a person he/she meets. The system was used to elicit user behaviors and feedback in realistic interaction tasks. We find that users do need information to aid their interactions. They are more inclined to use Google Glass to access information than a traditional mobile phone. By delving into the potential value of the system and the causes of negative feelings, we elaborate on the implications of the technology on user experience. With proper strategies to align the technical capabilities with user needs, it seems possible to exploit the technology to augment humans in social interactions.

\section{ACKNOWLEDGEMENTS}

The work is funded by the Singapore A*STAR JCO Visiting Investigatorship Programme - Revers-Engineering Visual Intelligence for Cognitive Enhancement (Project No. 1335h00098).

\section{REFERENCES}

1. Denning, T., Dehlawi, Z. and Kohno, T. In situ with bystanders of augmented reality glasses: Perspectives on recording and privacy-mediating technologies. in Proc. CHI'14, (2014), 2377-2386.

2. DeVaul, R.W., Pentland, A. and Corey, V.R. The memory glasses: Subliminal vs. overt memory support with imperfect information. in Proc. ISWC'03, (2003), 146-153.

3. Fiske, S.T. and Taylor, S.E. Social Cognition: From Brains to Culture. McGraw-Hill, 2007.

4. Hong, J. Considering privacy issues in the context of google glass. Commun. ACM, (2013), 10-11.

5. Iwamura, M., Kunze, K., Kato, Y., Utsumi, Y. and Kise, K. Haven't we met before? - A realistic memory assistance system to remind you of the person in front of you. in Proc. AH'14, (2014), No.32.

6. Kalnikaite, V., Ehlen, P. and Whittaker, S. Markup as you talk: Establishing effective memory cues while still contributing to a meeting. in Proc. CSCW'12, (2012), 349-358.

7. Kane, S.K., Linam-Church, B., Althoff, K. and McCall, D. What we talk about: Designing a context-aware communication tool for people with aphasia. in Proc. ASSETS'12, (2012), 49-56.

8. Lydon, J.E., Jamieson, D.W. and Zanna, M.P. Interpersonal similarity and the social and intellectual dimensions of first impressions. Social Cognition. 6, 4, (1988), 269-286.

9. Mandal, B., Chia, S.C., Li, L., Chandrasekhar, V., Tan, C. and Lim, J.H., A wearable face recognition system on google glass for assisting social interactions. in Proc. $A C C V^{\prime} 14,(2014)$

10. McNancy, R., Vines, J., Roggen, D., Balaam, M., Zhang, P., Poliakov, I. and Olivier, P. Exploring the acceptability of google glass as an everyday assistive device for people with Parkinson's. in Proc. CHI'14, (2014), 2551-2554.

11. Mulvenna, M., Martin, S., Sävenstedt, S., Bengtsson, J., Meiland, F., Dröes, R., Hettinga, M., Moelaert, F. and Craig, D. Designing \& evaluating a cognitive prosthetic for people with mild dementia. in Proc. ECCE'10, (2010), 11-18.

12. Tentori, M. and Hayes, G.R. Designing for interaction immediacy to enhance social skills of children with autism. in Proc. UbiComp'10, (2010), 51-60.

13. Utsumi, Y., Kato, Y., Kunze, K., Iwamura, M. and Kise, $\mathrm{K}$. Who are you? - A wearable face recognition system to support human memory. in Proc. AH'13, (2013), 150-153. 